\title{
Free Molecular Gas Drag on Fluffy Aggregates
}

\author{
R. Nakamura \\ The Graduate School of Science and Technology,Kobe University, Nada, \\ Kobe 657, Japan
}

\begin{abstract}
Computer simulations of the collisions of gas molecules with fluffy aggregates have been carried out to estimate gas drag forces in the free molecular flow limit. It is found that the gas drag force on fluffy aggregates can be approximated by that on an area-equivalent sphere in a wide range of relative velocities of the aggregate through ambient gas. The deviation from this approximation is weakly dependent on the relative velocity. This result leads to a significant increase of gas drag forces on fluffy aggregates compared to that on a mass-equivalent sphere.
\end{abstract}

\section{Introduction}

It has been generally recognized that coagulation of solid small particles leads to the formation of fluffy aggregates, rather than compact spheres. We frequently encounter astrophysical situations where the mean free path of the gas molecules is much longer than the size of the aggregate. Analytical gas drag formulae in the free molecule regime, available only for some simple shape with convex geometry(Dahneke 1973), yield the well known result that the drag force is proportional to the projected area of the target. Thus, most of the previous literature (e.g.,Weidenschilling \& Cuzzi 1993) simply assumed that the drag force on fluffy aggregate is proportional to the projected area as well. The main objective with this paper is ta examine this intuitive conjecture.

\section{Numerical Models}

Meakin et al. (1989) investigated the drag force on fractal aggregates in the Epstein regime where the relative velocity of an aggregate through the ambient gas is much smaller than the mean thermal velocity of the gas. On the other hand, our previous work (Nakamura et al. 1994) treated an aggregate moving much faster than the mean thermal velocity. Both are based on the assumption that the gas drag force can be expressed as the product of the following two independent factors.

1. the number of molecular collisions per unit time,

2. the mean momentum transfer per collision 
While the former is supposed to be proportional to the relative velocity and the projected area of the aggregate, the latter depends on the surface structure of the aggregate and also on the model for the molecular-particle interaction. Since it is difficult to estimate the value of the above factors for fluffy aggregates, previous researchers resorted to Monte-Carlo simulations (Meakin et al. 1989, Nakamura et al. 1994). They made independent estimates of the two factors and simply combined them, neglecting a possible correlation between them. Although each result quantitatively confirmed the conjecture that the gas drag force on fractal aggregates can be estimated by that on a sphere of equivalent geometrical cross section, the validity of the basic assumption that the drag force can be separated into two factors is still an open question. Therefore, we have developed new models to simulate the specular reflections of incident molecules on the surface of the aggregate. The correlation between the rate of molecular collision and the mean momentum transfer is taken into account explicitly in this scheme. Mulivariate integrations were performed using the Quasi Monte-Carlo method ( Niederreiter 1978). Details of these calculations will be published elsewhere (Nakamua 1995, in preparation). Two kinds of target aggregates are considered in this article, i.e., BPCA (Ballistic Particle Cluster Aggregate) and TTS (Two-Touching Spheres). TTS denotes an aggregate consisting of two identical spheres in contact. For the details of the generation processes and the resulting structures of BPCA, we refer to Mukai et al. (1992)

\section{Results and Discussions}

In contrast to the case of a single sphere, the fluffy aggregates have a large anisotropy in their shape. Thus, two directions of the motion are selected to represent extreme cases. One is the direction along the axis with the maximum momentum of inertia of the aggregate ( $\mathrm{X}$ direction), and another is that for the minimum ( $Z$ direction). Roughly speaking, these directions give the largest and smallest projected area of the aggregate, respectively.

Since the aggregate contains a large amount of vacuum, many molecules fired to the virtual sphere enclosing the aggregate miss the target. Figure 1 shows the collision probability, i.e., the fraction of impinging molecules that experienced at least one collision. The horizontal axis $\mathrm{S}$ denotes a ratio of the relative velocity to the mean thermal velocity of the gas. While the fraction is independent of the direction when $\mathrm{S}<<1$, anisotropy becomes clear as $\mathrm{S}$ increases. For TTS, two representative directions correspond to the perpendicular motion, in which it translates orthogonally to the line of centers, and the parallel motion along the line of centers, respectively. Since the radius of the enclosing sphere is twice as that for a constituent sphere, collisional probability becomes 0.5 for the $\mathrm{X}$ direction and 0.25 for the $\mathrm{Z}$ direction in the high velocity limit.

The product of this collision probability and the surface area of an virtual sphere enclosing the target aggregate can be interpreted as the effective surface area of the target when $\mathrm{S}<<1$. Further, this quantity completely agrees with the projected area of the target estimated by Meakin and Donn (1988) and Ossenkopf (1993) as the molecular motion approaches one way flow with increasing 


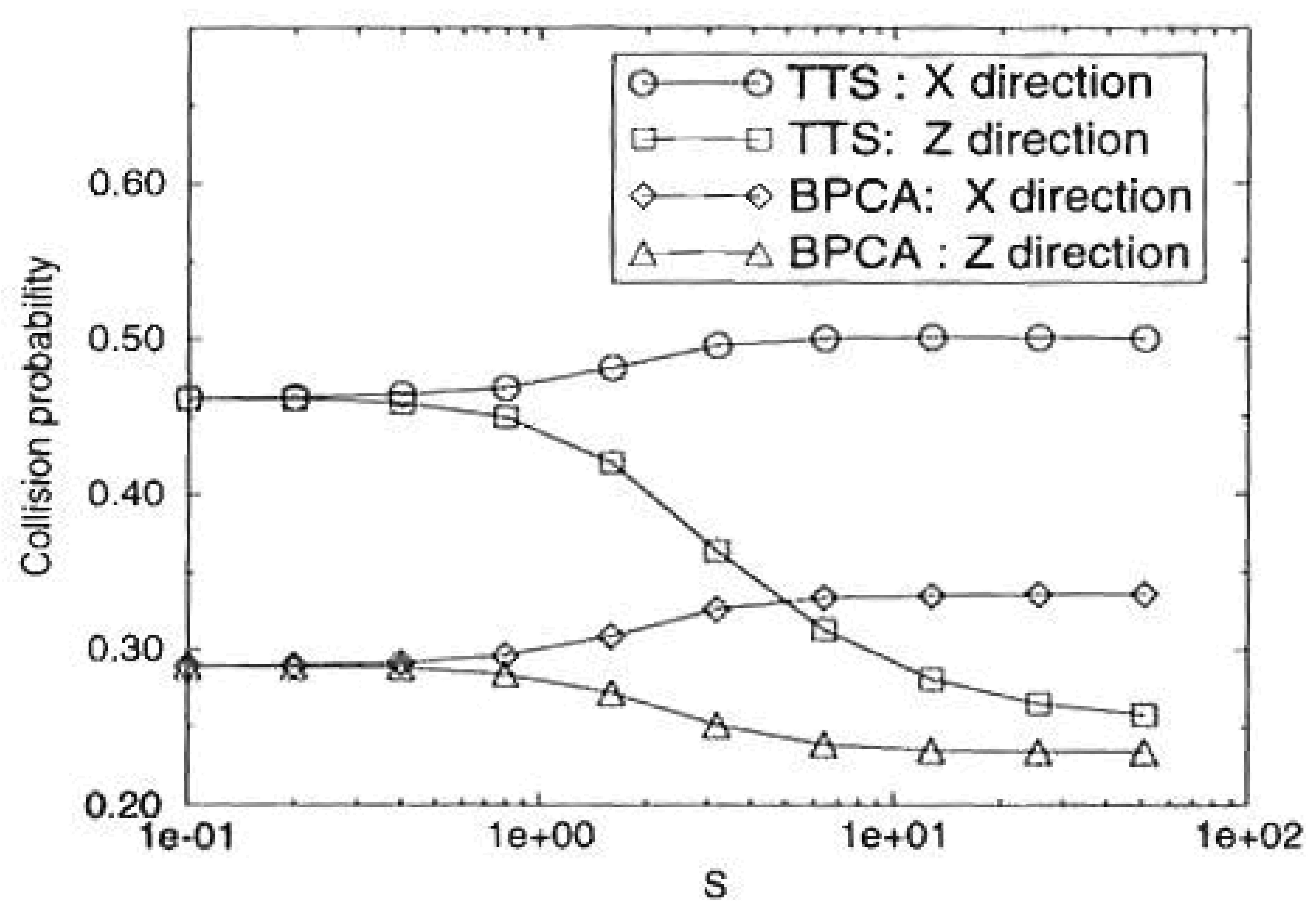

Figure 1. Fraction of incident molecules that experienced at least one collision. The horizontal axis represents $\mathrm{S}$, the velocity of the aggregates normalized by the mean thermal velocity of the gas. The targets are two-touching spheres (TTS) and BPCA with 8 constituent particles.

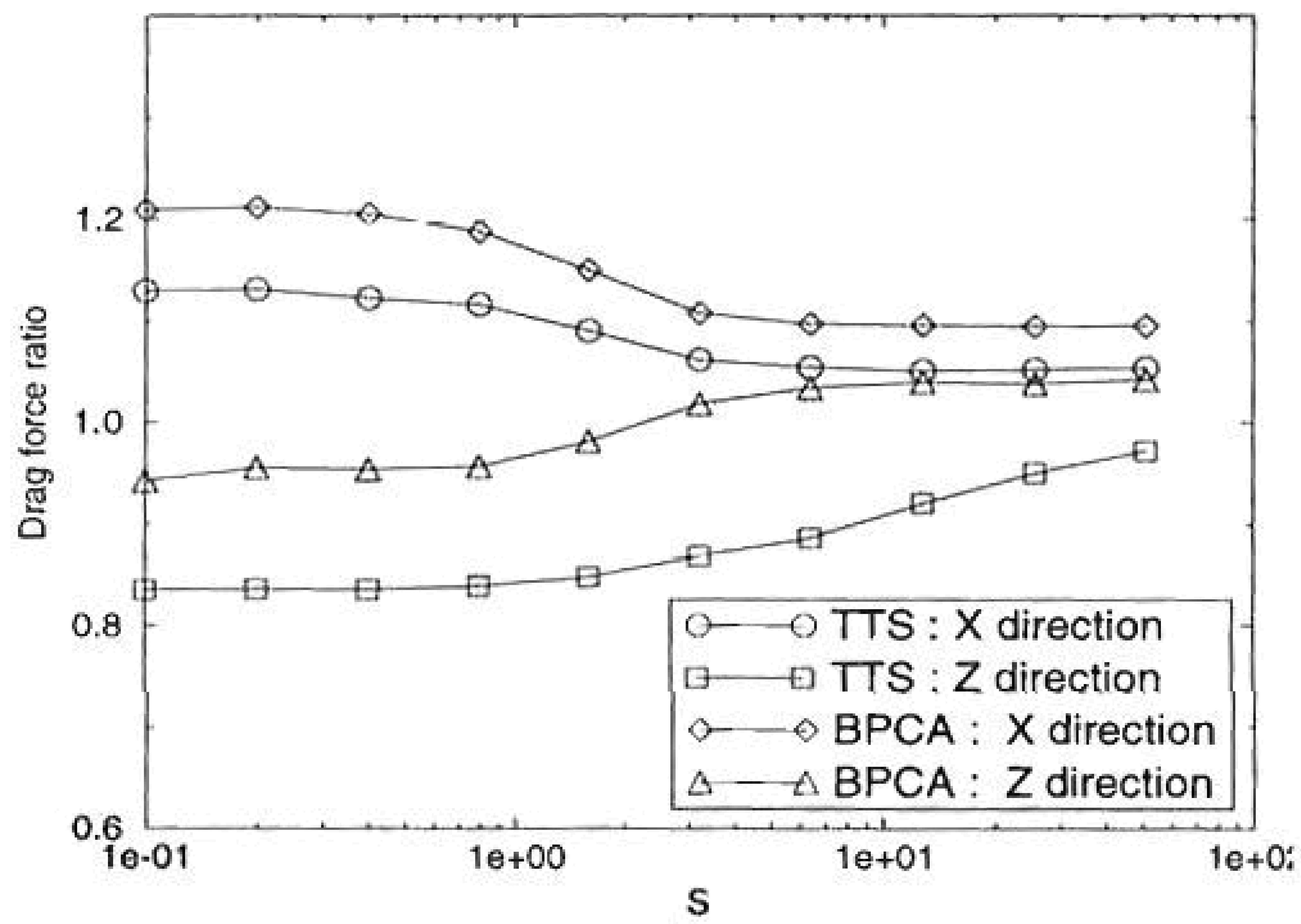

Figure 2. Same as Fig.1, but for the ratios of actual drag force on the target to that on an "area equivalent sphere". 
S. When the collision probability in the high velocity limit is averaged for three directions, it is nearly the same as that for $\mathrm{S}<<1$. If we adopt this 'mean' collision probability globally, however, the resulting drag forces reflect the variation of the collision probability depending on the direction of motion. In order to eliminate this effect, the local collision probability at a certain value of $\mathrm{S}$ is adopted to determine the 'area-equivalent sphere'.

In Fig. 2, the ratios of drag force on TTS and BCCA to that on an areaequivalent sphere are indicated. It should be emphasized that the ratio indicates anticorrelation with the collision probability. As a result of this cancelation, the variation of the drag force ratio becomes less distinct if the actual drag is globally normalized by the collision probability for $\mathrm{S}<<1$. It is revealed that the ratio depends on the size and the structure of the aggregate, but less than $25 \%$ for the entire range of S. Further, one can see from Fig. 2 that the drag force differs considerably even when $\mathrm{S}<<1$ and the collision probability is nearly same. Hence, the actual drag force depends on the direction of motion, not only for $\mathrm{S}>>1$ where the shape anisotropy appears, but also for $\mathrm{S}<<1$. In conclusion, our results quantitatively show the validity and the limitation of an intutitive conjecture that drag forces on fluffy aggregates are proportional to the projected area. The drag force on large aggregates will be investigated in our futuer work. Recently, Blum et al. (1995) measured the diffusion constants of fluffy agggregates in a microgravity environment. It is possible to derive the drag coefficient from the Einstein relation for those aggregates. Their preliminary results seem to support our theoretical prediction.

\section{References}

Blum, J., private commucation

Dahneke, B. E., 1973, Aerosol Sci,. 4, 147

Meakin, P., B. Donn, and G. W. Mulholland , 1989, Langmuir 5, 510

Mukai, T.,H. Ishimoto, T. Kozasa,J. Blum and J. M. Greenberg, 1992, A\&A 262,315

Nakamura, R. Kitada,Y. and Mukai,T., 1994, Planet. Space Sci. 42, 721

Niederreiter, H., 1978, Bull. Am. Math. Soc., 84, 957

Ossenkopf, V., 1993, A\&A 280, 617

Weidenschilling, S. J. and J. N. Cuzzi, 1993, in : Protostars and Planets III, eds. E. H. Levy, J. I. Lunine, University Arizona press p. 333 